\title{
Cannabis sativa e sua influência na capacidade de inteligência de seus usuários
}

\section{Cannabis sativa and its influence on intelligence of its users}

\author{
Fabiano de Abreu Agrela Rodrigues, \\ Logos University International, USA (deabreu.fabiano@gmail.com)
}

\begin{abstract}
Resumo: Popularmente conhecida como maconha ou marijuana, Cannabis sativa apresenta compostos que podem ser utilizados como terapêuticos e substâncias que afetam o sistema nervoso e, devido a tal fato, é classificada como uma droga ilícita. A principal substância psicoativa da cannabis demonstra enorme influência no cérebro, o THC atinge o sistema nervoso central, reconhece receptores no local, se liga e essa ação é responsável por seus efeitos. Assim, o objetivo do presente estudo é compreender se indivíduos que fazem o uso recreativo da Cannabis sativa podem ser afetados na diminuição da inteligência e, portanto, parte-se de uma revisão de literatura, realizada por meio das seguintes bases de dados: SciELO, PubMed PsycINFO e Science Direct. Com o uso crônico da substância é possível desenvolver alguns efeitos maléficos na atenção, na função cognitiva, memória operacional e tais efeitos em conjunto dificultam o aprendizado do indivíduo.
\end{abstract}

Palavras-Chave: Cannabis Sativa, Maconha, Inteligência, Memória, Sistema Nervoso.

\begin{abstract}
Popularly known as marijuana, Cannabis sativa presents compounds that can be used as therapeutics and substances that affect the nervous system and, due to this fact, it is classified as an illegal drug. The main psychoactive substance in cannabis shows enormous influence on the brain, THC reaches the central nervous system, recognizes receptors in the area and binds, and this action is responsible for its effects. Thus, the aim of this study is to understand whether individuals who make recreational use of Cannabis sativa can be affected by decreased intelligence and, therefore, it starts from a literature review, carried out through the following databases: SciELO, PubMed PsycINFO and Science Direct. The chronic use of the substance is possible to develop some harmful effects on learning, attention, cognitive function and working memory and such effects together hinder the individual's learning.
\end{abstract}

Keywords: Cannabis Sativa, Marijuana, Intelligence, Memory, Nervous System. 


\section{INTRODUÇÃO}

\subsection{Planta Cannabis sativa}

Popularmente conhecida como maconha ou marijuana, Cannabis sativa apresenta compostos considerados terapêuticos e substâncias que afetam o sistema nervoso e, devido a esse fato, é classificada como uma droga ilícita. Deriva de uma planta que apresenta mais de 400 substâncias químicas, das quais, algumas delas têm efeitos medicinais como o CBD e outras, psicoativas como THC (ElSohly et al., 2017). Sua principal maneira de administração é por meio da inalação (fumada), método que leva a um efeito rápido no organismo, estimando-se que, em cerca de meia hora, atinja os níveis máximos no sangue. Outra maneira de administração é ingerindo-a (Volkow et al., 2014).

Seu consumo remonta a partir do terceiro milênio a.C. e, atualmente é utilizada para recreação ou fins terapêuticos (ElSohly et al., 2017) e em 2723 a.C os chineses utilizavamna em sua farmacopéia. Do norte da China Central imigrou para Índia, Oriente Médio e chegou à Europa no final do século XVIII e início do século XIX. Com a colonização africana houve trocas de escravos que faziam uso da maconha até ser introduzida no Brasil. Nas grandes navegações, confeccionava-se lonas, velas e cordas a partir das fibras da maconha. Até ao século XX, a maconha era utilizada como planta medicinal e suas fibras no mercado textil, e até aos anos 40 ela fazia parte da farmacopéia oficial de diversos países, mas, a partir desse século e dos estudos de inúmeros cientistas, o seu uso foi desconsiderado e até proibido devido aos efeitos colaterais e narcodependência.

Segundo a Organização das Nações Unidas (ONU), cerca de quatro por cento da população mundial (162 milhões de pessoas) já utilizou ao menos uma vez a cannabis e cerca de 0,6 por cento (22,5 milhões) fazem o uso diariamente. No Brasil, segundo a Secretaria Nacional de Políticas sobre Drogas, na Cartilha sobre maconha, cocaína e inalantes a cada 100 indivíduos, 9 já a utilizaram uma vez, sendo a maioria, homens e na faixa de 18 a 24 anos de idade (Dhadwal et al., 2018).

Em Abril de 2019, o governo brasileiro aprovou a PNAD em Decreto 9.761. tendo em vista o disposto na Lei $\mathrm{n}^{\circ} 11.343$, de 23 de agosto de 2006. Tal política apresenta dados estatísticos atuais sobre o consumo no país conscientizando a população sobre os malefícios do uso de substâncias psicoativas. Além disso, ela diferencia os indivíduos que fazem uso dos indivíduos que realizam o comércio e traz inúmeras orientações para a conscientização, o combate e o tratamento da dependência química como um problema de saúde pública.

As substâncias químicas encontradas na Cannabis relevam potenciais terapêuticos (Corrêa, et al., 2020). Para usos terapêuticos, na década de 1990, houve a descoberta do primeiro mediador endógeno, denominado anandamida e o lançamento do primeiro medicamento atuante no sistema de endocanabinoides, denominado rimonabanto, que tinha como finalidade reduzir o apetite e tratar a obesidade (Cohen, Weizman, e Weinstein, 2019). Em 2019, a Agência Nacional de Vigilância Sanitária regulamentou os requisitos para medicamentos com Cannabis através da Resolução RDC no 327/19. Atualmente, a aplicação clínica do sistema de canabinoides possui maior conhecimento e atua na redução dos sintomas motores da Doença de Parkinson, na dor neuropática ou inflamatória, dentre outras. A FDA nos EUA aprovou tratamento coadjuvante à base de canabidiol para epilepsia e Parkinson, o que também pode ser liberado no Brasil, mediante prescrição 
médica, quando os outros medicamentos não se mostram mais eficazes, principalmente nos casos da epilepsia e, apesar de não serem fabricados no país.

Os efeitos imediatos da cannabis são: relaxamento, leve euforia, diminuição passageira na memória de curto prazo, boca seca, habilidades motoras levemente debilitadas e vermelhidão dos olhos. Os efeitos neurológicos de curto prazo mais comuns são: aumento da frequência cardíaca e apetite, diminuição da memória de curto prazo, coordenação psicomotora e da concentração. Dentre os efeitos a longo prazo são: danos respiratórios quando inalada (Volkow et al., 2014).

\subsection{Tetra-hidrocanabinol (THC)}

Dentre as mais de 400 substâncias, a principal substância psicoativa da cannabis que demonstra enorme influência no cérebro é o THC (Figura 1) que, ao atingir o sistema nervoso central, reconhece receptores no local e se liga, sendo essa ação a responsável por seus efeitos como por exemplo, na memória, na falta de coordenação, alucinógenos dentre outros (Dinis-Oliveira, 2016). Ao final do século XX, na década de 90, a revelação do Sistema Endocanabinoide (SECB) trouxe novas perspectiva para o estudo da substância em aplicações terapêuticas e discussão sobre a licitude ou não de seu uso. Tal sistema, pode resumidamente ser explicado como um sistema neuromodulatório onipresente que objetiva a homeostase corporal, integrando e modulando várias funções como rítmo circadiano, apetite, digestão, imunidade, plasticidade, dor, metabolismo, proteção neural, dentre outros.

Figura 1 - Composição química do THC (Fonte: Adaptado de Fogaça et al. 2020)<smiles>CCCCCc1cc(O)c2c(c1)OC(C)(C)[C@@H]1CCC(C)=C[C@H]21</smiles>

Quando absorvido na corrente sanguínea, as concentrações de THC diminuem rapidamente devido ao metabolismo do fígado, fazendo com que a depuração plasmática atinja $950 \mathrm{~mL} / \mathrm{min}$. A substância permanece em maior parte no tecido adiposo (devido à sua grande lipofília) obtendo seu pico de concentração em 4-5 dias. Após isso, é liberado lentamente atingindo as demais áreas do cérebro (Dinis-Oliveira et al., 2016). No cérebro, é distribuído de diversas maneiras, atingindo altos níveis de concentração nas áreas neocortical, límbica, sensorial e motora.

No fígado é metabolizado CYP 450 essencialmente a 11-hidróxi-THC (potencialmente mais potente que o THC), porém o 11-hidroxi-THC pode ainda ser substrato do álcool desidrogenase. Entre 10 13 dias, 25\% dos metabolitos são excretados por meio da urina sob a forma de éster de ácido glucurónico. A maioria da excreção é 
liberada no intestino através do fígado, podendo ser reabsorvido (circulação enterohepática), aumentando sua ação. Também pode ser eliminado por meio das fezes verificando a predominância de maneira não conjugada. O processo de eliminação total pode demorar até 30 dias. Graças à sequestração e à existência de metabolitos ativos, é difícil compreender a relação entre a concentração de THC no plasma ou urina e a intoxicação (Bindesri et al., 2020).

Substâncias análogas ao $\Delta 9$-THC, quimicamente modificadas permitiram o reconhecimento dos receptores canabinoides CB1 e CB2 (Cannabinoid binding receptor type 1 e type 2). São denominados endocanabinóides produzidos pelo organismo humanos a partir do consumo dos ácidos graxos. O primeiro, CB1 encontrado no SNC, em áreas que controlam a aprendizagem, a memória, as funções cognitivas superiores e o equilíbrio energético, é responsável pelos principais efeitos dos canabinoides, ou seja, os efeitos psicotrópicos, enquanto o CB2 fica a cargo dos efeitos periféricos: nos músculos, órgãos e tecidos expressando-se principalmente no sistema imunológico, na micróglia. ${ }^{1}$ Os receptores canabinoides pertencem à família dos receptores vinculados à proteína $\mathrm{Ge}$ vinculam- se, com as proteínas Gi e interagem com proteínas Gs ou Gq. (Paronis et al., 2012).

Os dois receptores vinculados a uma proteína que, quando ativada, inibe a enzima adenilato ciclase, aumenta a atuação dos canais de potássio e inibe canais de cálcio, coordenando a liberação de outros neurotransmissores (Castillo, et al., 2012). O receptor CB1 é amplamente identificado em várias estruturas encefálicas, especialmente em regiões vinculadas ao controle motor, como no centro da base e no cerebelo. $\mathrm{O}$ estriado ventral também possui grande densidade e intermedia os efeitos hedônicos e reforçadores dos canabinoides. A densidade dos receptores CB1 também é considerável no hipocampo e em outras áreas do córtex cerebral. (Djeungoue- Petga \& Hebert-Chatelain, 2017).

Os receptores CB1 e CB2 influenciam diversos neurotransmissores como por exemplo, o GABA, glutamato, noradrenalina, serotonina e dopamina, essa liberada no núcleo accumbens, potencializando as suas ações afetando diretamente na cognição, na percepção, no funcionamento motor, apetite, sono, neuroproteção proteção neural, neurodesenvolvimento e liberação hormonal. (Devinsky et al. 2014).

\subsection{Sistema nervoso}

O Sistema Nervoso é fundamental para o funcionamento do corpo na realização das atividades de locomoção, raciocínio e memória. Formado por neurônios e células da glia, pode ser classificado em sistema nervoso central (SNC) e sistema nervoso periférico (SNP). O sistema nervoso central é constituído por duas partes básicas: o encéfalo e a medula espinhal. O encéfalo contido no interior da caixa craniana e a medula espinhal encontra-se no interior da coluna vertebral, no canal vertebral (Gibbons et al., 2019).

O sistema nervoso periférico está conectado com as outras partes do corpo humano fora do SNC e é formado por fibras (nervos), gânglios nervosos e órgãos terminais. Suas demais funções são: enviar informações aos órgãos receptores sensoriais, responsáveis pela visão, audição, olfato, paladar e tato, até o SNC e trazer resposta adequada deste para os órgãos certos (Heiss \& Olofsson 2019).

\footnotetext{
${ }^{1}$ As micróglias são células neuronais centrais que se destacam por sondar o tecido cerebral e a medula.
} 


\section{OBJETIVO}

Compreender se o uso recreativo da Cannabis sativa, pode influenciar na diminuição da inteligência de indivíduos que a utilizem.

\section{METODOLOGIA}

O presente estudo é uma revisão de literatura, realizada por meio das seguintes bases de dados: SciELO, PubMed PsycINFO e Science Direct e foram utilizados os seguintes termos para realização da busca de dados em português: Cannabis sativa, maconha, inteligência, neurociência, memória, sistema nervoso e em inglês: Cannabis sativa, marijuana, intelligence, neuroscience, memory, nervous system.

Foram entrevistados cinco psicólogos de maneira anônima cujo requisito pricipal era possuir pacientes que fizessem o uso crônico da Cannabis como fumo, a fim de compreender se há perda da memória, dificuldade de aprendizagem, atenção e alterações nas funções cognitivas.

\section{RESULTADOS}

Por meio de entrevista com os cinco psicólogos, foi relatado que seus pacientes crônicos usuários da Cannabis afirmaram ter dificuldade no aprendizado apresentando perda de memória, dificuldades em permanecer concentrados em determinada função e alterações nas funções cognitivas. Um dos psicólogos entrevistados, relatou: "O comportamento de usuários contínuos é de lentidão, falta de memória, falta de localização espacial, perda de motivação, falta de raciocínio lógico, comportamento disfuncional para a ordem prática da vida. Não mantêm relacionamentos, não têm engajamento social ou acadêmico, não conseguem ter grande sucesso profissional, são dependentes e solicitantes. Mantêm um comportamento infantilizado mesmo quando em idade adulta".

$\mathrm{O}$ uso da Cannabis promove efeitos imediatos no indivíduo: esse apresenta indisposição para realizar algo, vermelhidão nos olhos, a boca seca, a visão e audição distorcidas, o sistema imunológico prejudicado, os batimentos cardíacos ficam acelerados, dando uma sensação de euforia, seguida de relaxamento e riso fácil. Devido ao seu uso prolongado, o homem pode-se tornar infértil, pois diminui a quantidade de testosterona produzida, responsável pelas características masculinas, tais como voz grossa, barba, músculos e produção de espermatozóides (Ford et al., 2017).

Devido ao uso contínuo, por meio da neuroimagem, verificou-se sérios danos nos ventrículos laterais e do terceiro ventrículo, desenvolvendo atrofia cortical (Block et al., 2000).

No estudo de Wilson et al. (2000) com 57 indivíduos, sendo 32 homens e 25 mulheres, avaliou-se o volume cerebral dos seguintes itens: cérebro total, volumes dos ventrículos laterais e substância branca e cinzenta, demonstrando que indivíduos que utilizavam maconha antes dos 17 anos em comparação àqueles que começaram depois, apresentaram uma menor percentagem de substância cinzenta cortical, com maior diferença nos lobos 
frontais. Em relação à substância branca cerebral, houve uma maior percentagem d22e substância branca cerebral nos indivíduos que fizeram o uso durante a adolescência.

\subsection{Cannabis e seus efeitos na função cognitiva}

O uso da Cannabis também influencia no aprendizado, na memória, na atenção e na memória operacional (Grant et al., 2003). Um ensaio clínico controlado encontrado em duas meta-análises, demonstrou que usuários apresentaram pior desempenho em testes neuropsicológicos e nas funções executivas específicas, na atenção, no aprendizado e memória, nas habilidades motoras e verbais, em comparação ao grupo não-usuários (Schreiner et al., 2012).

Estudos realizados com adolescentes e adultos usuários por meio de neuroimagem, demonstraram alterações estruturais em regiões temporais mediais (amígdala e hipocampo), frontais e cerebelares associadas à exposição ao uso da cannabis (Lorenzetti et al., 2014; Batalla et al., 2014). Outros estudos científicos apontam que o uso regular da Cannabis em pacientes com idade inferior aos 20 anos, pode resultar em déficits cognitivos, desordens psiquiátricas e aumento significativo da probabilidade de dependência da substância, pois, o sistema neurológico ainda se encontra em formação, tendo o núcleo accumbens e área tegumentar ventral (centro de prazer) altamente desenvolvido e a região do córtex pré-frontal, responsável pelo juízo crítico ainda em desenvolvimento (Ribeiro, 2014)

Zalesky et al. (2012) avaliou o comprometimento da conectividade neural de adultos usuários da Cannabis os quais apresentaram diminuição na conectividade na fímbria direita do hipocampo (fórnix) e do esplênio do corpo caloso, e nas fibras comissurais.

\subsection{Danos na memória}

Atualmente, existem evidências que usuários de maconha podem sofrer problemas no processamento da memória a curto prazo. A hipótese é de que a Cannabis compromete a sinalização neural quando se ligam aos receptores responsáveis pela memória no cérebro podendo afetar a capacidade de aprendizagem ocorrendo problemas de concentração. A memória é dividida em memória de curto e longo prazo. A memória de curto prazo mantém temporariamente e a memória de longo prazo é onde as informações são guardadas indefinidamente (Hamilton \& Hughes, 2020).

Um estudo realizado por Schweinsburg et al. (2008) com 15 adolescentes usuários de maconha, mostrou anormalidades na resposta do cérebro em relação ao grupo controle de 12 indivíduos. Os mesmos testes para memória foram repetidos após 1 mês de abstinência e mesmo assim não houve diferenças em relação à avaliação anterior.

Um ensaio clínico através do exame de ressonância magnética, realizado por Yucel et al. (2008) com adultos com média de idade de 20 anos, que faziam uso diário da cannabis, comparado ao grupo que não fazia tal uso demonstrou que o grupo usuário da droga, apresentou alterações cerebrais estruturais, expostas na diminuição de $7 \%$ no volume da amígdala e $12 \%$ de diminuição nos volumes bilaterais do hipocampo.

\subsection{Dificuldades de atenção, organização e integrar informação complexa}


Sabe-se que o uso crônico da maconha acarreta em dificuldades de atenção, organização, memória e compreensão de informações complexas. Num estudo com 24 usuários, em que 12 participantes passaram por um mês de abstinência, foi demonstrado que após esse período, os efeitos do uso crônico da droga podem ser reversíveis. Entretanto, essa reversibilidade ainda é incerta sendo necessárias mais pesquisas, não é possível confirmar que a maconha não possa provocar alterações nas redes corticais ou que essas alterações não ocorram, pois, o cérebro se adapta às mudanças induzidas pela droga. Por meio de testes de urina positivos para THC ficou provado maior ativação nas regiões cerebelares frontal e medial, nos usuários ativos ao uso do que aqueles em abstinência, havendo um maior uso da rede de reserva, ou seja, regiões com efeito de carga, sugerindo um estado neuroadaptativo ${ }^{2}$ (Chang et al., 2006).Em um estudo realizado por Lundqvist (2005) foi relatado que usuários de maconha demonstram dificuldades em codificar informações na memória de longo prazo, aprendizagem verbal prejudicada, sendo assim se distraem com maior facilidade, tendo aumento de tais efeitos com a continuidade do uso da droga.

\subsection{Efeitos psicóticos}

O uso crônico da maconha é conhecido como fator de risco para a psicose. Em relação à associação da Cannabis e a esquizofrenia, pode variar suas causas como: genéticoambientais, etiologias compartilhadas ou da automedicação para sintomas pré-mórbidos (Ferraro et al., 2013). Efeitos psicóticos são alucinações tais como, ouvir vozes, ver pessoas ou objetos que não existem, ter a certeza que está sendo perseguido por alguém ou por câmeras (Miettunen et al., 2008).

Isso se deve ao fato de que o THC, um dos principais componentes da maconha, quando ingerido em doses altas pode causar psicose aguda, transitória e dependente da dose (sintomas positivos e negativos similares aos esquizofrênicos) (Miettunen et al., 2008). Em pacientes com patologias psicóticas, os canabinoides podem aumentar os sintomas resultando em consequências negativas ao longo do desenvolvimento da doença. Quanto mais precoce a idade de contato com a substância, maior é a probabilidade de desenvolver dependência e doenças de cunho reprodutivo, neurológico, imunológico, entre outros, podendo inclusive ocasionar prejuízos funcionais limitantes, da perspectiva produtiva, social e familiar (Aizpurua-Olaizola et al., 2014, Radhakrishnan et al., 2014).

\section{Considerações finais}

A maconha (Cannabis sativa) é a droga ilícita mais utilizada em todo o mundo, apesar de não ter tanta importância como outras drogas, cocaína, crack e até o álcool devido ao fato de que seus efeitos são mais subtis que as demais. Com seu uso crônico, é possível desenvolver alguns efeitos maléficos como, por exemplo, no aprendizado, atenção, função cognitiva e memória operacional.

\footnotetext{
${ }^{2}$ Estado Neuroadaptativo pode ser caracterizado pela tolerância ou sensibilização comportamental diante do uso repetitivo e compulsivo de determinada substância psicoativa, por exemplo. A sensibilização comportamental implica em adaptações neuroquímicas e moleculares do sistema dopaminérgico. (Robinson, Becker, 1986; Nestler, Hyman, Robert, 2001).
} 
O fato do indivíduo não conseguir se concentrar na leitura de um livro, assistir a uma vídeo-aula e também não conseguir memorizar tais aprendizagens deve-se ao fato de que a maconha causa efeitos no processamento da memória a curto prazo. Tais conjuntos de efeitos dificultam o aprendizado do indivíduo. Com o tempo de uso, a região frontal do cérebro relacionada à inteligência, encontra-se prejudicada, com atrofia (Martin-Santos et al., 2010) e os neurônios se moldam nesta condição resultando em uma inteligência pobre o que pode trazer problemas para a vida diante de situações que exijam o raciocínio lógico e tomada de decisões.

O uso da maconha causa transtornos de ordem mental e psiquiátrica, a depender da propensão genética desencadeando esquizofrenia e outros distúrbios psicóticos e transtornos afetivos. Esses distúrbios surgem como conseqüência da disfunção na região límbica do cérebro e diminuição da região da inteligência.

Ao prejudicar a memória de curto prazo, no hipocampo, dificulta o armazenamento da memória de longo prazo, já que esta região é quem condiciona o armazenamento definitivo de memória. Esta alteração pode ser irreversível mesmo com treinamentos cognitivos na tentativa de desenvolver as regiões afetadas mediante a uma plasticidade cerebral.

\section{Referências}

Batalla, A.; Crippa, J.A.; Busatto, G.F.; Guimaraes, F.S.; Zuardi, A.W.; Valverde, O.; Atakan, Z.; McGuire, P.K.; Bhattacharyya, S.; Martín-Santos, R. (2014). Neuroimaging Studies of Acute Effects of THC and CBD in Humans and Animals: a Systematic Review. Current Pharmaceutical Design, 20(13). 2168-2185(18).

Bindesri, S. D., Jebailey, R., Albarghouthi, N., Pye, C. C., \& Brosseau, C. L. (2020). Spectroelectrochemical and computational studies of tetrahydrocannabinol (THC) and carboxy-tetrahydrocannabinol (THC-COOH). Analyst, 145(5), 1849-1857. https://doi.org/10.1039/C9AN02173F

Block, R. I., O'Leary, D. S., Hichwa, R. D., Augustinack, J. C., Ponto, L. L. B., Ghoneim, M. M., ... \& Andreasen, N. C. (2000). Cerebellar hypoactivity in frequent marijuana users. Neuroreport, 11(4), 749-753.

Castillo, P. E., Younts, T. J., Chávez, A. E., \& Hashimotodani, Y. (2012). Endocannabinoid signaling and synaptic function. Neuron, 76(1), 70-81. https://doi.org/10.1016/j.neuron.2012.09.020

Chang, L., Yakupov, R., Cloak, C., \& Ernst, T. (2006). Marijuana use is associated with a reorganized visual-attention network and cerebellar hypoactivation. Brain, 129(5). 1096-1112. https://doi.org/10.1093/brain/awl064

Cohen, K., Weizman, A., \& Weinstein, A. (2019). Positive and negative effects of cannabis and cannabinoids on health. Clinical Pharmacology \& Therapeutics, 105(5), 1139-1147. https://doi.org/10.1002/cpt.1381

Corrêa, L. T., Plata, C. F., Ricci, E. L., Nicoletti, M. A., Caperuto, E. C., de Souza Spinoza, H., ... \& Fukushima, A. R. (2020). Revisão Bibliográfica Sistematica- 
Sistema de Endocanabinoides Tendências de Uso na Farmacologia. Brazilian Journal of Forensic Sciences, Medical Law and Bioethics, 9(2), 146-167.

https://doi.org/10.17063/bjfs9(2)y2020146

Decreto No 9.761, de 11 de Abril (2019). Aprova a Política Nacional sobre Drogas.

Diário Oficial da União: Edição 70-A, Seção 1 Extra, Página

https://www.in.gov.br/materia/-

/asset publisher/Kujrw0TZC2Mb/content/id/71137357

Devinsky, O., Cilio, M. R., Cross, H., Fernandez-Ruiz, J., French, J., Hill, C., ... \& Friedman, D. (2014). Cannabidiol: pharmacology and potential therapeutic role in epilepsy and other neuropsychiatric disorders. Epilepsia, 55(6), 791-802. https://doi.org/10.1111/epi.12631

Dhadwal, G., \& Kirchhof, M. G. (2018). The Risks and Benefits of Cannabis in the Dermatology Clinic. Journal of Cutaneous Medicine and Surgery, 22(2), 194-199. https://doi.org/10.1177/1203475417738971

Dinis-Oliveira, R. J. (2016). Metabolomics of $\Delta 9$-tetrahydrocannabinol: implications in toxicity. Drug metabolism reviews, 48(1), 80-87. https://doi.org/10.3109/03602532.2015.1137307

Djeungoue-Petga, M. A., \& Hebert-Chatelain, E. (2017). Linking mitochondria and synaptic transmission: the CB1 receptor. Bioessays, 39(12), 1700126. https://doi.org/10.1002/bies.201700126

ElSohly, M. A., Radwan, M. M., Gul, W., Chandra, S., \& Galal, A. (2017). Phytochemistry of Cannabis sativa L. Phytocannabinoids, 1-36. https://doi.org/10.1007/978-3-319-45541-9_1

Ferraro, L., Russo, M., O'Connor, J., Wiffen, B. D., Falcone, M. A., Sideli, L., ... \& Di Forti, M. (2013). Cannabis users have higher premorbid IQ than other patients with first onset psychosis. Schizophrenia Research, 150(1), 129-135. https://doi.org/10.1016/j.schres.2013.07.046

Fogaça, J.R.V. (2020) THC - Principal componente ativo da maconha. Mundo Educação. https://bit.ly/3qszTFQ

Ford, T. C., Hayley, A. C., Downey, L. A., \& Parrott, A. C. (2017). Cannabis: an overview of its adverse acute and chronic effects and its implications. Current drug abuse reviews, 10(1), 6-18. https://doi.org/10.2174/1874473710666170712113042

Gibbons, C. H. (2019). Basics of autonomic nervous system function. Handbook of clinical neurology, 160, 407-418. https://doi.org/10.1016/B978-0-444-64032-1.00027-8

Grant, I., Gonzalez, R., Carey, C. L., Natarajan, L., \& Wolfson, T. (2003). Non-acute (residual) neurocognitive effects of cannabis use: a meta-analytic study. Journal of the International Neuropsychological Society, 9(5), 679-689. https://doi.org/10.1017/S1355617703950016

Hamilton, I., \& Hugues, E. (2020, julho 16). Maconha faz mal - mas também faz bem - à memória. Projeto Colabora. https://bit.ly/3HagGhY 
Heiss, C. N., \& Olofsson, L. E. (2019). The role of the gut microbiota in development, function and disorders of the central nervous system and the enteric nervous system. Journal of neuroendocrinology, 31(5), e12684. https://doi.org/10.1111/jne.12684

Lorenzetti, V., Solowij, N., Fornito, A., Ian Lubman, D., \& Yucel, M. (2014). The association between regular cannabis exposure and alterations of human brain morphology: an updated review of the literature. Current pharmaceutical design, 20(13), 2138-2167.

Lundqvist, T. (2005) Cognitive consequences of cannabis use: comparison with abuse of stimulants and heroin with regard to attention, memory and executive functions. Pharmacol Biochem Behav. 81(2), 319-330 https://doi.org/10.1016/j.pbb.2005.02.017

Martín-Santos, R., Fagundo, A., Crippa, J., Atakan, Z., Bhattacharyya, S., Allen, P., . . . McGuire, P. (2010). Neuroimaging in cannabis use: A systematic review of the literature. Psychological Medicine, 40(3), 383-398. https://doi.org/10.1017/S0033291709990729

Miettunen, J., Törmänen, S., Murray, G., Jones, P., Mäki, P., Ebeling, H., . . Veijola, J. (2008). Association of cannabis use with prodromal symptoms of psychosis in adolescence. British Journal of Psychiatry, 192(6), 470-471. https://doi.org/10.1192/bjp.bp.107.045740

Nestler, E. J.; Hyman S. E.; Robert, C. M. (2001). Molecular neuropharmacology: a foundation for clinical neuroscience. New York: McGraw-Hill. 539p.

Paronis, C. A., Nikas, S. P., Shukla, V. G., \& Makriyannis, A. (2012). 49 Tetrahydrocannabinol acts as a partial agonist/antagonist in mice. Behavioural pharmacology, 23(8), 802. https://doi.org/10.1097\%2FFBP.0b013e32835a7c4d

Radhakrishnan, R., Wilkinson, S. T., \& D'Souza, D. C. (2014). Gone to pot-a review of the association between cannabis and psychosis. Frontiers in psychiatry, 5, 54. https://doi.org/10.3389/fpsyt.2014.00054

Ribeiro, J. A. C. (2015). A cannabis e suas aplicações terapêuticas. Tese de Doutorado. Universidade Fernando Pessoa. http://hdl.handle.net/10284/4828

Robinson, T. E., \& Becker, J. B. (1986). Enduring changes in brain and behavior produced by chronic amphetamine administration: a review and evaluation of animal models of amphetamine psychosis. Brain research reviews, 11(2), 157-198. https://doi.org/10.1016/0165-0173(86)90002-0

Schreiner, A. M., \& Dunn, M. E. (2012). Residual effects of cannabis use on neurocognitive performance after prolonged abstinence: a meta-analysis. Experimental and clinical psychopharmacology, 20(5), 420-429. https://doi.org/10.1037/a0029117

Schweinsburg, A. D., Nagel, B. J., Schweinsburg, B. C., Park, A., Theilmann, R. J., \& Tapert, S. F. (2008). Abstinent adolescent marijuana users show altered fMRI response during spatial working memory. Psychiatry Research: Neuroimaging, 163(1), 40-51. https://doi.org/10.1016/j.pscychresns.2007.04.018 
Volkow, N. D., Baler, R. D., Compton, W. M., \& Weiss, S. R. (2014). Adverse health effects of marijuana use. New England Journal of Medicine, 370(23), 2219-2227. https://doi.org/10.1056/NEJMra1402309

Wilson, W., Mathew, R., Turkington, T., Hawk, T., Coleman, R. E., \& Provenzale, J. (2000). Brain morphological changes and early marijuana use: a magnetic resonance and positron emission tomography study. Journal of addictive diseases, 19(1), 1-22. https://doi.org/10.1300/J069v19n01_01

Yücel, M., Solowij, N., Respondek, C., Whittle, S., Fornito, A., Pantelis, C., \& Lubman, D. I. (2008). Regional brain abnormalities associated with long-term heavy cannabis use. Archives of general psychiatry, 65(6), 694-701. https://doi.org/10.1001/archpsyc.65.6.694

Zalesky, A., Solowij, N., Yücel, M., Lubman, D. I., Takagi, M., Harding, I. H., ... \& Seal, M. (2012). Effect of long-term cannabis use on axonal fibre connectivity. Brain, 135(7), 2245-2255. https://doi.org/10.1093/brain/aws136 